\title{
Hydraulic contacts controlling water flow across porous grains
}

\author{
A. Carminati, ${ }^{1, *}$ A. Kaestner, ${ }^{1}$ H. Flühler, ${ }^{1}$ P. Lehmann, ${ }^{2}$ D. Or, ${ }^{2}$ E. Lehmann, ${ }^{3}$ and M. Stampanoni ${ }^{4}$ \\ ${ }^{1}$ Institute of Terrestrial Ecosystems, ETH Zurich, Switzerland \\ ${ }^{2}$ Laboratory of Soil and Environmental Physics, EPF Lausanne, Switzerland \\ ${ }^{3}$ Spallation Neutron Source Division, Paul Scherrer Institut, Villigen, Switzerland \\ ${ }^{4}$ Swiss Light Source, Paul Scherrer Institut, Villigen, Switzerland
}

(Received 19 October 2006; revised manuscript received 22 June 2007; published 21 August 2007)

\begin{abstract}
Water flow between porous grains varies widely depending on the water distribution in contacts between grains. The hydraulic behavior of contacts varies from highly conductive when water fills the contacts to a bottleneck to flow as water pressure drops and contact asperities rapidly drain. Such changes greatly impact the hydraulic conductivity of porous grain packs such as aggregated soil. The dominant driving force of water flow across contacts is capillarity, often quantified relative to gravity and viscous forces using the capillary and Bond numbers. For fast water infiltration, viscous forces dominate. For simplicity we modeled the water distribution between spherical porous grains whose surfaces are covered by spherical bumps of much smaller radii. We provide experimental evidence obtained by neutron radiography and synchrotron-based x-ray tomographic microscopy documenting transitions in the flow behavior across contacts.
\end{abstract}

DOI: 10.1103/PhysRevE.76.026311

\section{INTRODUCTION}

Variably saturated porous media are ubiquitous in nature. Water flow occurs at many scales ranging from millimeters to kilometers. Water fluxes are essential for sustaining biological activity, nutrient cycling, and numerous other processes in the unsaturated zone between soil surfaces and aquifers and have a myriad of applications in industry and engineering. In this study we focus on water flow in media composed of porous grains or aggregates that are partially saturated with water. Media composed of porous grains are common in topsoils [1], pavement and construction materials, and pharmaceutical applications. This study is also applicable to other flow processes, such as thermal, electric, and solute fluxes, in granular materials [2].

The movement of water through partially saturated porous media is driven by gravity, capillary, and viscous forces. Depending on the relevant importance of these forces, different flow behaviors occur [3]. The relative magnitude of the forces is expressed by the dimensionless capillary $(\mathrm{Ca})$ and Bond (Bo) numbers:

$$
\begin{aligned}
& \mathrm{Ca}=\frac{\mu V r^{2}}{\sigma \kappa}, \\
& \mathrm{Bo}=\frac{\rho g r^{2}}{\sigma},
\end{aligned}
$$

with $\mu$ being the fluid viscosity, $V$ the fluid velocity, $r$ the pore radius, $\sigma$ the surface tension, $\kappa$ the intrinsic permeability of porous medium, $\rho$ the fluid density, and $g$ gravity. Ca expresses the relative magnitude of viscosity and capillarity, and Bo quantifies the relative value of gravity and capillarity. Depending on the flow conditions and pore radius, extremely different flow regimes occur [3]. In case of low flow rates

\footnotetext{
*Present address: Department of Hydrogeology, UFZ Leipzig, Germany.
}

PACS number(s): 47.56.+r, 47.80.Jk, 68.35.Ct, 81.05.Rm

and small pores, capillary forces are dominant and $\mathrm{Ca} \ll 1$. When water is displaced by a nonwetting fluid such as air (drainage), capillarity can induce a fingering regime $[4,5]$. At high flow rates, viscous forces dominate capillarity and $\mathrm{Ca}$ $\gg 1$. The effect of viscosity depends on which phase is the most viscous and dense [6]. When water is displaced by air (drainage), viscosity increases the flow instability. When air is displaced by water (imbibition), viscosity has a stabilizing effect. In large pores gravity becomes more significant and the Bond number Bo increases. Gravity stabilizes the wet front during drainage and destabilizes the flow field during imbibition from the top. The relative importance of the driving forces causes the transition between the different flow behaviors, which modifies the macroscopic flow and transport properties [7].

Flow patterns become even more complex due to the inherent heterogeneity of porous media. Small-scale heterogeneities, structures, fissures, and capillary barriers may control the global flow process at different scales. The existence of various pore classes, such as macropores and micropores, present a challenge to univocally defining $\mathrm{Ca}$ and $\mathrm{Bo}$.

The role of heterogeneities in media composed of porous grains is illustrated in Fig. 1, showing the infiltration of water through two vertically stacked porous grains. Drops of water were injected onto the top grain, and the water movement into the second grain was monitored by means of neutron radiography [8]. The grains are natural aggregates of $5 \mathrm{~mm}$ in diameter collected from the surface horizon of a forrest soil. Depending on small variations in the volume of injected water, two distinct cases can be observed.

Case 1. If the quantity of the applied water is sufficient to saturate the contact between the two grains, the wet front rapidly moves throughout the second grain.

Case 2. If less water is applied, the water front stops at the contact and the flow process becomes much slower. The transition between case 1 and case 2 is abrupt.

Our hypothesis is that this flow behavior is caused by a sudden drainage of pores in the contact between grains. These contact pores are likely larger than the pores within the individual grains and are therefore drained more easily at 

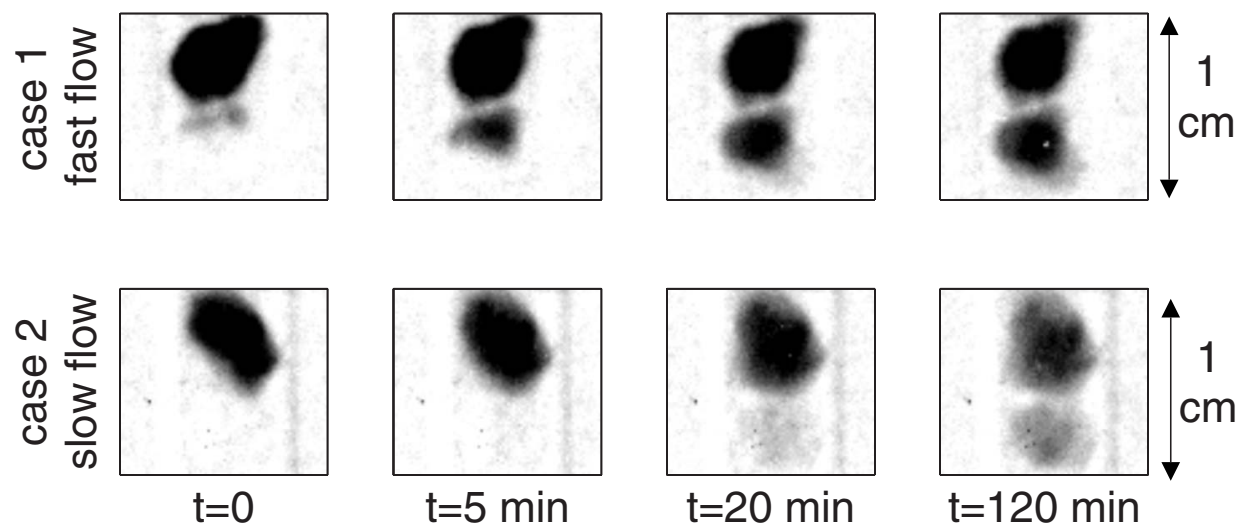

FIG. 1. Neutron radiographs of water infiltration through soil grains (aggregates) stacked vertically. A few drops of water were added onto the top aggregate. Depending on small variations in the volume of added water, two cases can occur: a fast infiltration (case 1) or a much slower water transfer. The gray values are proportional to the thickness of water in the beam direction. low tension. The existence of large contact pores is due to the roughness of the grains. A porous surface is notoriously rough [9]. It has been described with a fractal approach [10-12]. Halsey and Levine [13] discussed the importance of the contact pores on the mechanical stability of granular media. Despite the fact that knowledge and a description of rough surfaces are well established, the role of the contact roughness on the water flow through porous grains has received little attention.

The objective of this study is to describe the physics of the water flow across the contacts. We intend to evaluate the relative importance of capillary, viscous, and gravity forces at differnet flow rates. In addition, we propose a model to describe the water distribution in the contacts and the hydraulic conductivity of a series of aggregates. To this end we used x-ray microscopic tomography to image the distribution of air, water, and solids in the contact region at varying water potential. Based on this information and data from Fig. 1, we calculated the hydraulic conductivity of water flow between grains and the capillary and Bond numbers characterizing this hydraulic system.

The present article is organized as follows: in Sec. II we model the hydraulic behavior of the contact region in terms of the flow cross section and hydraulic conductance, which is the product of the flow cross section times hydraulic conductivity. From the local properties of the contacts we up-scale to define the macroscopic conductivity of a series of grains. In Sec. III we describe the materials and methods used to image the water distribution in the contact region. In Sec. IV we present the results from x-ray tomography in comparison with the proposed model. Then we calculate the capillary number $\mathrm{Ca}$ and the Bond number Bo for the observed water exchanges (Fig. 1). In Sec. V we discuss the importance of the contacts on the flow process and the relative importance of the involved forces. Finally we propose potential applications of this work.

\section{CONCEPTUAL MODEL}

\section{A. General concepts: Hydraulics of unsaturated media}

In the following, we review some general concepts of water flow in unsaturated porous media. Water is retained in porous media by capillarity and adsorptive forces that act at the solid surfaces of the porous matrix. These forces can be expressed in potential form as the matric potential $\psi_{\mathrm{m}}$, which is the energy required to bring a unit volume of water into the porous medium $\left[\mathrm{J} \mathrm{m}^{-3}\right]$. In the present article we express the matric potential as matric head, $h=\rho g \psi_{\mathrm{m}}$, with $\rho$ being the density of water and $g$ gravity. When the amount of adsorbed water is a negligible fraction of the total water, $h$ is related to the curvature of the air-water interface by the classical Young-Laplace equation $h=-\frac{2 \sigma}{\rho g r}$, where $\sigma$ is the surface tension and $r$ is the average curvature radius of the gas-liquid interface. Varying $h$ modifies the volume of water retained in the pore space. It is well known that the conductivity of unsaturated porous media, $k\left[\mathrm{~m} \mathrm{~s}^{-1}\right]$, varies with $h$-i.e., $k(h)$ [14].

In media composed of microporous grains, most of the water is retained by capillarity within the grains and the large intergranular pores are air filled. Under these conditions water moves through the network of connected grains. It is a flow in series through grains and contacts, the latter either being conductive or acting as bottlenecks. This situation is similar to the inverted Swiss-cheese model $[15,16]$, also referred to as inverted random void system. The inverted Swiss-cheese model is a medium with randomly placed spherical voids embedded in an impermeable material. The liquid flows through the connected spherical voids. This flow process can be described using the percolation theory [16]. The basic idea is to assume that the conductivity is determined by the smallest cross section between adjacent spheres. The authors calculate the statistical distribution of the cross sections between randomly placed spheres and use percolation theory to determine the conductivity of the systems.

Our approach is different. We measure and model the flow cross section between adjacent grains, with explicit consideration of the pore geometry. The motivation is that the flow cross section does not depend only on the solid contacts between grains, which are the counterpart of voids in the inverted Swiss-cheese model. Instead, it depends in the first place on the saturation degree of the contacts. Indeed the flow cross section is given by the water-filled fraction of the contact area, which we refer to as $a_{\mathrm{w}}(h)$. Note that $a_{\mathrm{w}}$ decreases when the matric head $h$ and, consequently, the curvature radius of the gas-liquid interface are reduced. The function $a_{\mathrm{w}}(h)$ has not been properly measured and modeled so far. 


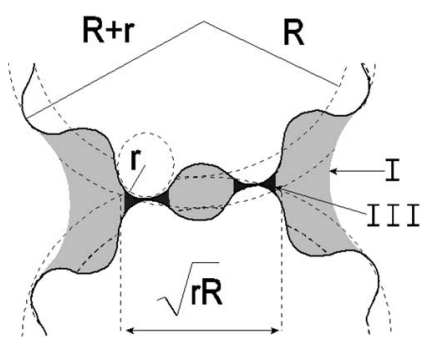

FIG. 2. Grains are described as large spheres covered with much smaller spheres. Under wet conditions a wide capillary bridge connects the large spheres (regime I). Under drier conditions air has invaded the asperities and water is stored in the capillary menisci between the small spheres (regime III). In the transition between regimes I and III, the water-filled contact area $a_{\mathrm{w}}(h)$ drastically decreases. In this figure the asperities are exaggerated and the contacts are illustrated in a very loose packing.

\section{B. Hydraulic properties of the contacts}

We propose a model to describe the hydraulic properties of such contacts: namely, the water-filled contact area $a_{\mathrm{w}}(h)$. We expect that $a_{\mathrm{w}}(h)$ is highly affected by the contact roughness. Under wet conditions, at high values of $h$, grains facing each other are hydraulically connected by a wide capillary bridge. The water-filled contact area $a_{\mathrm{w}}(h)$ is large, and the contacts are likely conductive. Under drier conditions, large voids in the contact region become suddenly air filled and the contacts are reduced to sparse and narrow hydraulic bridges. The water-filled contact area $a_{\mathrm{w}}(h)$ drops, and the contacts become much less conductive. The two hydraulic states of the contacts are illustrated in Fig. 2 and are referred to as regime I and III, respectively. Regime I likely leads to the fast flow illustrated in Fig. 1 (case 1), while regime III induces the slow flow (case 2). Regime II is the transition between the limiting cases I and III.

To properly model the behavior of $a_{\mathrm{w}}(h)$, the contact roughness must be modeled. Starting from the models of spheres [17-19], we describe a grain as a sphere of radius $R$ covered with much smaller spheres of radius $r$. We assume that the distance between the small spheres is $\sim r$. For such grains, $a_{\mathrm{w}}(h)$ can be divided into the three regimes which allow an analytical solution.

Regime I. Under wet conditions the surface asperities of the grains are water filled and a capillary bridge connects the external surface of the grains. The external surfaces have radius of $\sim R+r$ and overlap by $\sim r$. The area of the capillary bridge between such grains can be calculated with Eqs. $A 4$ A5 reported in Appendix A of [19]. This regime holds until the radius of curvature of the liquid-gas interface is larger than $r$-i.e., $h>-\frac{2 \sigma}{\rho g} \frac{1}{r}$.

Regime II. For $h<-\frac{2 \sigma}{\rho g} \frac{1}{r}$ the curvature radius of the liquid-vapor interface is smaller than the contact asperities and air invades the contact region. As air enters forming bubbles, the cross-sectional area $a_{\mathrm{w}}$ rapidly decreases. We assume for simplicity that for each step decrease of the radius of the liquid-gas interface from $r^{*}$ to $r^{*}-d r^{*}$, the decrease of $a_{\mathrm{w}}$ is constant. At the limiting value of $h=-\frac{2 \sigma}{\rho g} \frac{c}{r}$ all voids in the contact region are drained and the remaining water is stored in narrow capillary bridges between the asperities. The limit value $h=-\frac{2 \sigma}{\rho g} \frac{c}{r}$ corresponds to the drainage of a pore with radius $\frac{c}{r}$, which is supposed to be the smallest pore in the contact region. The estimation of $c \sim 10$ will be compared with data from x-ray tomography.

Regime III. For $h<-\frac{2 \sigma}{\rho g} \frac{c}{r}$ air has invaded the contact region and the remaining water is stored in the capillary bridges between the asperities, as shown in Fig. 2. The water-filled contact area is given by the pendular bridge between spheres of radius $r$. We assume no overlapping of the small spheres-i.e., a no-strain condition. The number of small spheres is calculated as the macroscopic contact area of the large spheres, approximately equal to $\pi r R$ [13], divided by the area of the small spheres, $\pi r^{2}$, which gives a number of contact spheres of $\sim R / r$.

\section{Hydraulic conductivity of a series of grains}

The water-filled contact area $a_{\mathrm{w}}(h)$ determines the conductivity of the contacts. We describe the properties of the contacts in terms of conductance, $K\left[\mathrm{~m}^{3} \mathrm{~s}^{-1}\right]$, which is defined as a conductivity $k\left[\mathrm{~m} \mathrm{~s}^{-1}\right]$ multiplied by the flow cross section $\left[\mathrm{m}^{2}\right]$. In its movement through the porous grains, water dissipates energy within the grains and the flow cross section in the contacts is equal to $a_{\mathrm{w}}(h)$. Considering this, the hydraulic conductance of the contacts is calculated as

$$
K_{\text {cont }}(h)=k_{\text {grain }}(h) a_{\mathrm{w}}(h),
$$

with $k_{\text {grain }}(h)$ being the conductivity of the grains.

Under unsaturated conditions, water flows through a series of grains and contacts. The equivalent equivalent conductance $K^{\mathrm{eq}}(h)$ is calculated as the harmonic mean of the conductances of the individual grains $K_{\text {grain }}(h)$ and their contacts $K_{\text {cont }}(h)$ :

$$
K^{\mathrm{eq}}(h)=\left(\frac{R}{K_{\text {grain }}(h)}+\frac{r}{K_{\text {cont }}(h)}\right)^{-1}(R+r),
$$

where $R$ and $r$ are the lengths of the flow path in the grain and in the contact, respectively. These lengths are deduced from the model shown in Fig. 2. $K_{\text {grain }}(h)$ is given by $k_{\text {grain }}(h)$ multiplied by the harmonic mean of the grain cross section. The values of $k_{\text {grain }}(h)$ and the grain cross sections were measured by means of flow simulation and neutron tomography [8].

\section{MATERIALS AND METHODS}

We used the technique of x-ray tomography to image the three-dimensional distribution of air, water, and solid in the contact region between grains at different matric heads $h$. We used soil aggregates from the same top soils as used in the experiment of Fig. 1.

Synchrotron-based x-ray tomographic microscopy (SRXTM) has been performed at the TOMCAT beamline of the Swiss Light Source located at the Paul Scherrer Institute (PSI), Switzerland. The spatial resolution of the microtomography setup was set to $5.92 \mu \mathrm{m}$, accommodating a field of view of $11 \mathrm{~mm}$. Two porous grains were placed into a Plexi- 

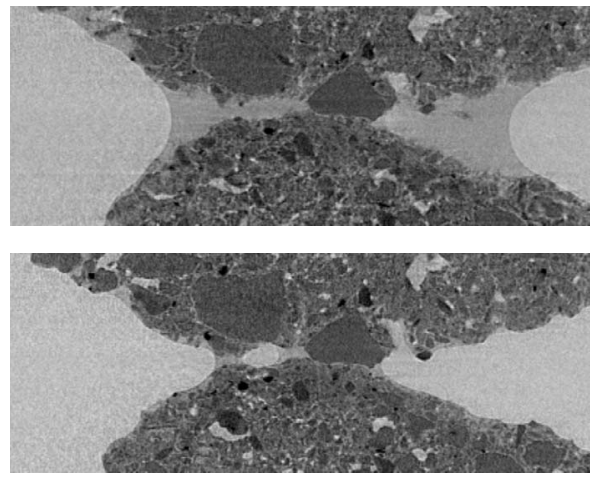

FIG. 3. Vertical section of the contacts between grains imaged by SRXTM. The sagittal view shows a $3 \times 1 \mathrm{~mm}^{2}$ part of the sample at a resolution of $5.92 \mu \mathrm{m}$. Top: the contacts at $h=$ $-0.025 \mathrm{~m}$. A wide capillary bridge connects the grains. Bottom: the sample at $h=-1.4 \mathrm{~m}$. Sparse and narrow menisci connect the grains.

glass cylinder which has a porous plate at the bottom to connect the grains to a water reservoir. The level of the water reservoir relative to the contact region corresponds to the matric head $h$ at the contacts. The sample was scanned at $h$ $=-0.025 \mathrm{~m}$ (wet conditions) and at $h=-1.4 \mathrm{~m}$ (drained condition). In Fig. 3 we show a vertical section of the contact region in these two conditions.

From the tomography data we calculated the water-filled contact area $a_{\mathrm{w}}$ for different matric heads $h$. The calculation is described in detail in [20]. We reconstructed the pore geometry by segmenting the tomogram in large pores and solids. Only pores larger than the spatial resolution were segmented while small pores are considered to be part of the solid grains. Then the water retained by capillarity in the segmented pore space was calculated with a morphological pore-network model [21]. The water distribution for varying $h$ is shown in Fig. 4. Then, we defined the contact surface: it is the middistance plane between facing grains. It separates the grains passing only through the contacts. The distribution of water in the contact surface gives the water-filled contact area $a_{\mathrm{w}}(h)$.

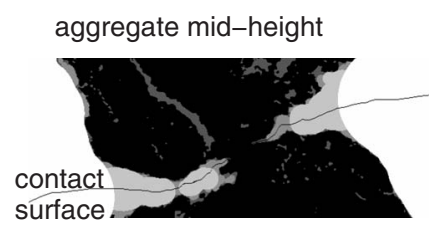

a)

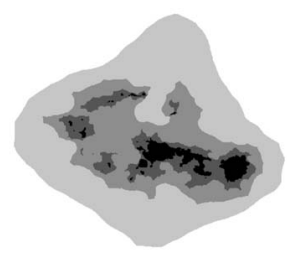

b)
FIG. 4. Calculated water distribution in the segmented pore space. Solids are black. The gray levels represent the distribution of water at different matric heads $h$. (a) Vertical section with the contact surface (line). (b) Water distribution in the contact surface viewed from the top.

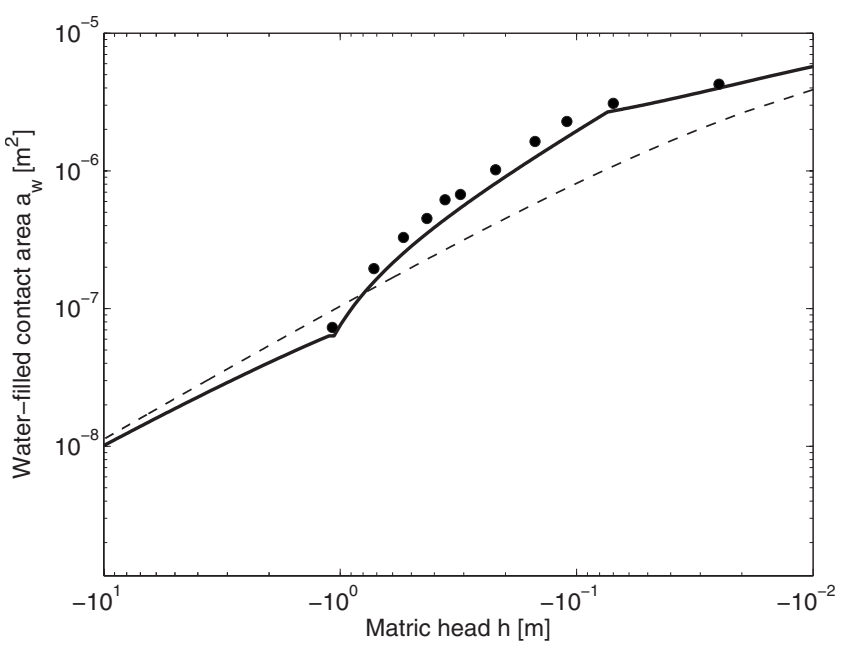

FIG. 5. Water-filled contact area $a_{\mathrm{w}}$ between grains as a function of the matric head $h$. The solid line corresponds to the rough grains, the dashed line to the model with smooth grains. The dots denote $a_{\mathrm{w}}(h)$ obtained from the tomographed soil grains.

\section{RESULTS}

\section{A. Water-filled contact area and conductivity}

The images from X-ray tomography (Fig. 3) show the contact region under wet and drier conditions. Under wet conditions (top), a wide capillary bridge connects the grains and the contacts are expected to be highly conductive. This situation corresponds to regime I of the model of rough spheres (Fig. 2). Under drier conditions (bottom), the contacts are air filled and the water-filled contact area is reduced to sparse and narrow capillary bridges between the surface asperities. The asperities are due to mineral particles and fissures on the grain surface. Under these conditions the contacts likely act as bottlenecks for the flow. This situation corresponds to regime III of our model.

The comparison between the water-filled contact area derived from x-ray tomography and predicted by the model is shown in Fig. 5. In Fig. 5 we plot $a_{\mathrm{w}}(h)$ for the tomographed grains (Fig. 4) and as predicted by the model with the parameters $R=2.5 \mathrm{~mm}, r=0.1 \mathrm{~mm}$, and $c=20$. The parameter $c$ is consistent with the value of $\sim 10$ predicted in Sec. II B. As a comparison, we plot also $a_{\mathrm{w}}(h)$ calculated for smooth spheres of $R=2.5 \mathrm{~mm}$ and $r=0 \mathrm{~mm}$. Under wet conditions, the model with rough surfaces gives a larger $a_{\mathrm{w}}(h)$. When $h$ decreases, the rough contacts are drained more rapidly than those between smooth spheres.

The modeled $a_{\mathrm{w}}(h)$ is used to calculate the conductance of the contacts, $K_{\text {cont }}(h)$ [Eq. (3)], and the equivalent conductance of series of aggregates, $K^{\mathrm{eq}}(h)[\mathrm{Eq}$. (4)]. The calculated conductances are plotted in Fig. 6 and compared with the conductance of the grain interior, $K_{\text {grain }}(h)$. Close to saturation the conductance of the contacts and that of the grains are similar. When the matric head $h$ decreases, $K_{\text {cont }}(h)$ falls rapidly, and at $h=-10 \mathrm{~m}, K^{\text {cont }} \approx 10^{-3} K_{\text {grain }}$. This is the effect of the decrease of $a_{\mathrm{w}}(h)$. The equivalent conductance of the sample $K^{\mathrm{eq}}(h)$ decreases as $K_{\text {cont }}(h)$ This indicates that the contacts effectively control the global conductivity of the entire system. 


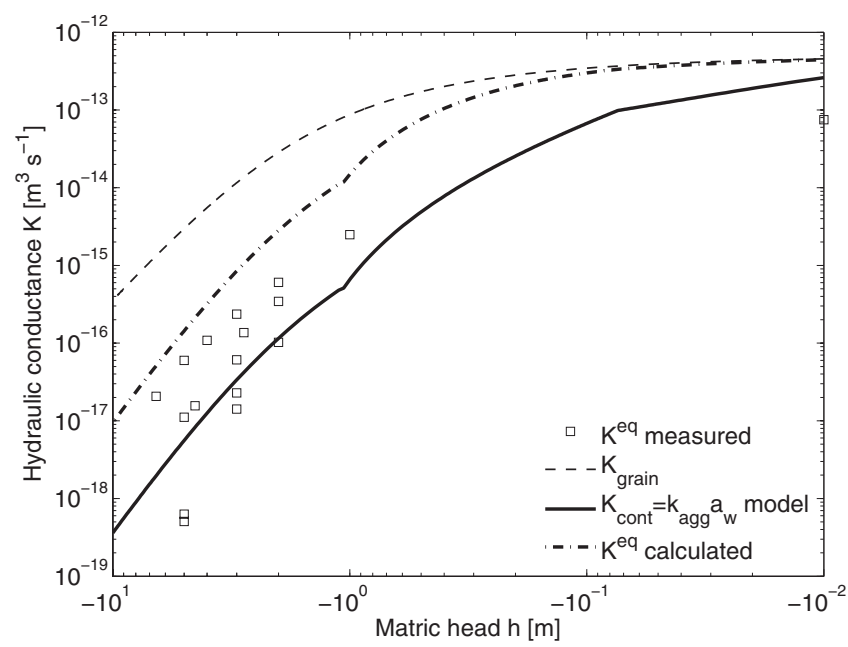

FIG. 6. Hydraulic conductance of series of grains, $K^{\mathrm{eq}}(h)$ $\left[\mathrm{m} \mathrm{s}^{-1}\right]$, compared to the conductance of the grain (aggregate) interior, $K_{\text {agg }}(h)$, and to that of the contacts, $K_{\text {cont }}(h) . K_{\text {cont }}(h)$ is calculated with Eq. (3), with $a_{\mathrm{w}}(h)$ obtained from the model of rough spheres. $K^{\mathrm{eq}}(h)$ is calculated with Eq. (4) and compared with independent measurements [8].

The calculated results are compared to experimental data obtained in previous experiments [8], where the water exchange between grains was quantified by means of neutron radiography and Darcy's law was used to determine the equivalent conductance. Measured and calculated $K^{\mathrm{eq}}(h)$ match well. In particular, the rapid decrease of $K^{\mathrm{eq}}(h)$ is reproduced very well.

\section{B. Capillary and Bond numbers}

In this section we calculate $\mathrm{Ca}$ and $\mathrm{Bo}$ for the water flow in 12 samples, named $B 6-B 15, A 1$ top and $A 1$ bottom (Table 2 in [8]).

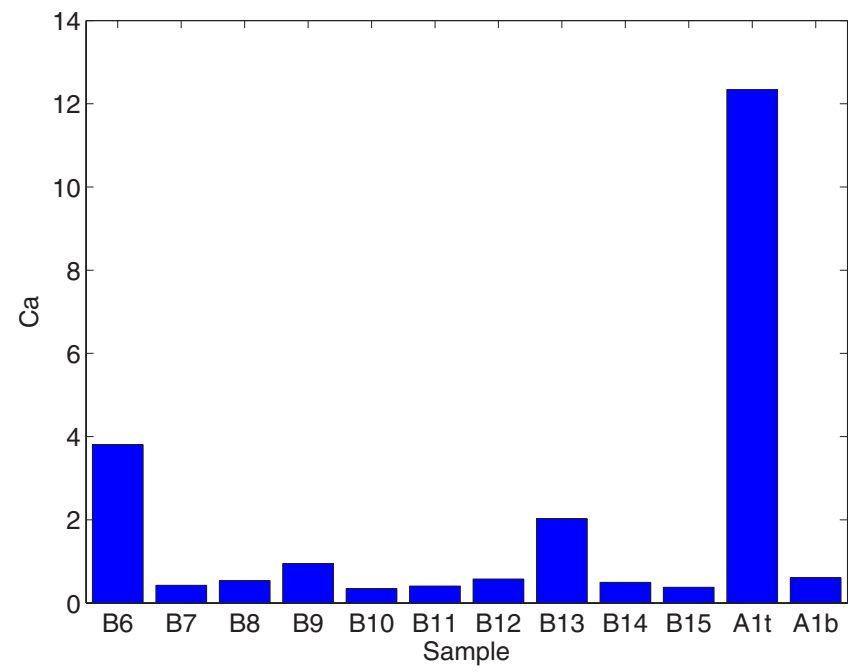

FIG. 7. (Color online) Capillary number of water flows from grain to grain, calculated for samples $B 6-B 15$ and $A 1$ (Table 2 in [8]). Fast water exchange was observed in samples $B 6, B 13$, and $A 1$ top, which have the highest values of $\mathrm{Ca}$. In these samples viscosity is important.
TABLE I. Capillary and Bond numbers for fast and slow flows between porous grains. Data are based on neutron radiography of water infiltration through soil porous grains of $5 \mathrm{~mm}$ in diameter.

\begin{tabular}{lcc}
\hline \hline & $\mathrm{Ca}$ & Bo \\
\hline Fast flow & $6.06 \pm 5.5$ & $0.051 \pm 0.034$ \\
Slow flow & $0.52 \pm 0.18$ & $0.002 \pm 0.001$ \\
\hline
\end{tabular}

$\mathrm{Ca}$ is defined in Eq. (1). $V$ is the liquid velocity in the contact and $r$ the pore radius in the contacts. The radius $r$ corresponds to the radius of the capillary bridge connecting the grains. Then we set $r^{2}=a_{\mathrm{w}} / \pi$. The product of $V$ with $r^{2}$ is the volume of water that flows across the contacts per unit time. This has been calculated as the amount of water that flows from grain to grain per unit of time. The term $\mu / \kappa$ is calculated as $\rho g / k$, where $k\left[\mathrm{~m} \mathrm{~s}^{-1}\right]$ is the conductivity of grains. For simplicity we assume that the micropores in the grain interior are water saturated and we set $k$ equal to the saturated value, $k=10^{-7} \mathrm{~m} \mathrm{~s}^{-1}$, as measured in [8]. $\sigma$ $=0.0728 \mathrm{~N} \mathrm{~m}^{-1}$ is the surface tension of water. The calculated values of $\mathrm{Ca}$ for the 12 samples are plotted in Fig. 7 (in the axis labels the samples $A 1$ top and $A 1$ bottom are named $A 1 t$ and $A 1 b$, respectively).

The highest values of $\mathrm{Ca}$ (see Table I) occur in the samples with fast water exchange ( $B 6, B 13$, and $A 1$ top). For these samples we obtained an average value of $\mathrm{Ca}$ $=6.06 \pm 5.5$, which indicates an important role of viscosity. For the samples showing a slow water exchange (case 2 of Fig. 2) we have an average value of $\mathrm{Ca}=0.52 \pm 0.18$. In this flow regime, viscosity becomes less important.

The Bond number is defined in Eq. (2). We used $r^{2}$ $=a_{\mathrm{w}} / \pi$, with the contact area calculated in Table 2 of [8]. The calculated values of Bo are plotted in Fig. 8. The samples with fast flow have $\mathrm{Bo}=0.051 \pm 0.034$, with a maximum value of 0.09 in sample $A 1$ top. The samples with slow flow have $\mathrm{Bo}=0.002 \pm 0.001$. In both fast- and slow-flow re-

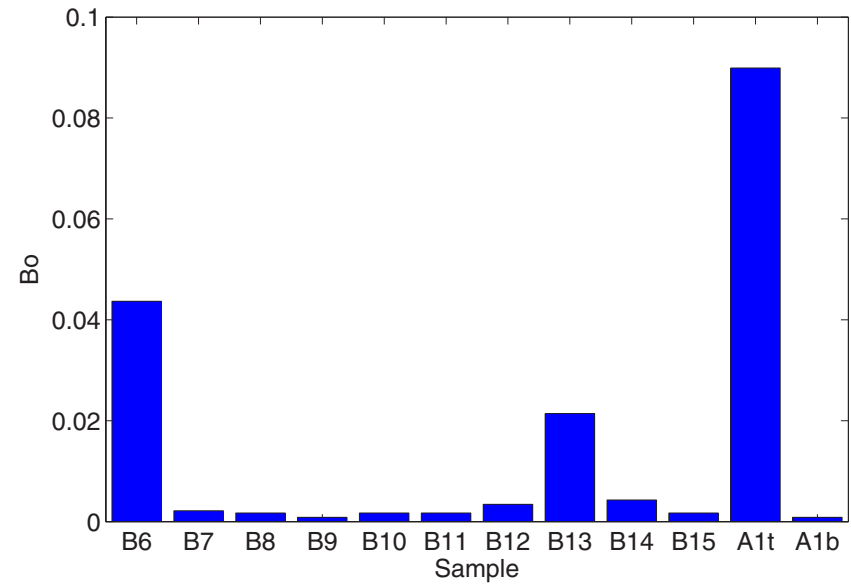

FIG. 8. (Color online) Bo of water flows for samples $B 6-B 15$ and $A 1$ (Table 2 in [8]). Bo values smaller than 0.1 indicate that capillarity is the most important driving force. Samples with fast water flow are characterized by larger Bo numbers, indicating an increasing relevance of gravity. 
gimes capillarity is the most important driving force. In fastflow regimes, gravity becomes relevant and it is approximately 20 times more important than in slow-flow regimes.

\section{DISCUSSIONS}

Water flow between porous grains can be very fast or extremely slow, depending on the water distribution in the grain-to-grain contacts. When the contact region is water filled, the contacts are highly conductive. As the water potential decreases, contact asperities become rapidly drained and the flow cross section drops. As a consequence the contacts become bottlenecks for the water flow, drastically reducing the conductivity of the system. We modeled this process describing a grain as a sphere covered by much smaller spheres. For such grains the water-filled contact area $a_{\mathrm{w}}(h)$ is divided in to three regimes that admit an analytical solution. From $a_{\mathrm{w}}(h)$ we derived the conductivity of the system. The model was validated by means of neutron and x-ray tomography data.

We found that the most relevant force driving the flow process is capillarity. In the case of slow water exchange, $\mathrm{Bo}=0.002 \pm 0.001$, indicating that gravity plays a minor role in this process. Gravity becomes more relevant in fast water regimes, where $\mathrm{Bo}=0.051 \pm 0.034$. However, capillarity remains the principal force. This indicates that the transition from fast to slow water flow is mainly due to the drainage of the capillary bridge between grains and consequent decrease of $a_{\mathrm{w}}(h)$. This justifies our description of the physical process in terms of $a_{\mathrm{w}}$ and matric potential.

The large variation of $a_{\mathrm{w}}$ as a function of the matric head $h$ causes the abrupt transition from the conductive to the bottleneck state of the contacts. From the experiment illustrated in Fig. 1, we observed that the transition between conductive and bottleneck states occurred for small variations in the applied volumes of water. This indicates the existence of a threshold in water content, and consequently in matric potential, which determines the switch from conductive to bottleneck states. This switch suggests the possibility to use percolation theory to model the flow through media composed of porous grains. The approach is different from that used in the inverted Swiss-cheese model, where the spatial distribution of the spheres determines the statistic of the conducting contact area. In our case the grains are assumed to be well connected in terms of solid contacts. The important point is the evolution of the matric head, which over a given threshold activates the contacts. This mechanism could be modeled with the invasion percolation approach [22]. Invasion percolation models assign to the sites of a network a probability distribution representing the unsaturated conductivity. Water primarily seeps through the site with a higher conductivity. Following a similar approach, we could describe the grains as nodes and the contacts as links which become active over a given matric potential. Imbibition processes could be then modeled and could give rise to fingering regimes.

This study can be extended to other fluxes of mass and energy. The relation between equivalent conductance and water-filled contact area can be applied to electric and thermal fluxes in granular materials. Since water is often the most conductive component of a system, the water-filled contact area $a_{\mathrm{w}}(h)$ is likely to be the key function for determining electric and thermal conductivities of wet granular media. Important applications are also in hydrology and agricultural science. Top soils are often composed of porous grains [1]. Under well-drained conditions, the contacts slow down the water flow, favor the water storage in the plant-root zone, and reduce evaporation by impeding the capillary rise. The prediction of these processes is of paramount importance due to the increasing demand for food and water.
[1] N. C. Brady and R. R. Weil, The Nature and Properties of Soils (Prentice-Hall, Upper Saddle River, NJ, 2002).

[2] H. M. Jaeger, S. R. Naegel, and R. B. Behringer, Rev. Mod. Phys. 68, 1259 (1996).

[3] Y. Méheust, G. Løvoll, K. J. Måløy, and J. Schmittbuhl, Phys. Rev. E 66, 051603 (2002).

[4] R. Chandler, J. Koplik, K. Lerman, and J. F. Willemsen, J. Fluid Mech. 119, 249 (1982).

[5] R. Lenormand and C. Zarcone, Transp. Porous Media 4, 599 (1989).

[6] G. M. Homsy, Annu. Rev. Fluid Mech. 19, 271 (1987).

[7] J. F. Chau and D. Or, Phys. Rev. E 74, 056304 (2006).

[8] A. Carminati, A. Kaestner, R. Hassanein, O. Ippisch, P. Vontobel, and H. Flühler, Adv. Water Resour. 30, 1168 (2007).

[9] B. Mandelbrot, D. Passoja, and A. Paullay, Nature (London) 308, 721 (1984).

[10] H. Pape, L. Riepe, and J. R. Schopper, J. Microsc. 148, 121 (1987).

[11] H. Pape, L. Riepe, and J. R. Schopper, Colloids Surf. 27, 97 (1987).

[12] D. Broseta, L. Barré, O. Vizika, N. Shahidzadeh, J.-P. Guil- baud, and S. Lyonnard, Phys. Rev. Lett. 86, 5313 (2001).

[13] T. C. Halsey and A. J. Levine, Phys. Rev. Lett. 80, 3141 (1998).

[14] W. A. Jury and R. Horton, Soil Physics (Wiley, Hoboken, NJ, 2004).

[15] B. I. Halperin, S. Feng, and P. N. Sen, Phys. Rev. Lett. 54, 2391 (1985).

[16] S. Feng, B. I. Halperin, and P. N. Sen, Phys. Rev. B 35, 197 (1987).

[17] W. Rose, J. Appl. Phys. 29, 687 (1958).

[18] C. D. Willet, M. J. Adams, S. A. Johnson, and J. P. K. Seville, Langmuir 16, 9396 (2000).

[19] T. M. Ghezzehei and D. Or, Water Resour. Res. 36, 367 (2000).

[20] A. Carminati, A. Kaestner, P. Lehmann, and H. Flühler (unpublished).

[21] P. Lehmann, P. Wyss, A. Flisch, E. Lehmann, P. Vontobel, M. Krafczyk, A. Kaestner, F. Beckmann, A. Gygi, and H. Flühler, Vadose Zone J. 5, 80 (2006).

[22] D. Wilkinson, Phys. Rev. A 34, 1380 (1986). 